Schlafbezogene Atmungsstörungen

\section{Häufigere Komplikationen in der Schwangerschaft}

Bourjeily G et al. Pregnancy and fetal outcomes of symptoms of sleep-disordered

breathing. Eur Respir J. 2010;36:849-855

\section{Hintergrund}

Physiologische Veränderungen während der Schwangerschaft begünstigen die Entstehung schlafbezogener Atmungsstörungen [1]. Die Häufigkeit des Neuauftretens von Schnarchen während bzw. infolge einer Schwangerschaft wird mit 14-45\% angegeben, wobei die Inzidenz nicht genau bekannt ist $[2,3]$.

Schlafbezogene Atmungsstörungen in der Schwangerschaft können einen Einfluss auf die Blutdruckregulation haben (Gestationshypertonie) [4]. Welcher Zusammenhang zu Stoffwechselstörungen wie Gestationsdiabetes besteht, ist dagegen unklar. Auch ist der Einfluss von schlafbezogenen Atmungsstörungen auf das Schicksal des Neugeborenen nicht abschließend beantwortet $[5,6]$.

\section{Fragestellung}

Die Studie untersuchte die Prävalenz schlafbezogener Atmungsstörungen in der Schwangerschaft und den Zusammenhang zu gesundheitlichen Komplikationen und dem Verlauf bei der Schwangeren und dem Neugeborenen.

\section{Methodik}

In einer Querschnittsuntersuchung von 1000 zufällig ausgewählten Frauen, die sich in der unmittelbaren postpartalen Phase befanden, wurde der multivariable Apnoe-Prädiktions-Index (MAPI) bestimmt. Die Anamnese sowie der Verlauf und Komplikationen der Schwangerschaft einschließlich der Art der Entbindung sowie die neonatale Entwicklung wurden erfasst. In der Regressionsanalyse wurden folgende Störvariablen berücksichtigt: Alter, Body Mass Index, Diabetes mellitus, chronische arterielle Hypertonie, Mehrlingsschwangerschaften, Rauchverhalten und Nierenerkrankungen. Das mittlere Alter der Frauen betrug 29,1 Jahre.

\section{Ergebnisse}

Schnarchen lag bei 35,1\% der Probandinnen vor. Frauen mit Symptomen schlafbezogener Atmungsstörungen wiesen ein gesteigertes Risiko für nachfolgende Verläufe, Erkrankungen bzw. Komplikationen auf:

- Gestationshypertonie/Präeklampsie (adjustierte Odds Ratio [OR] = 2,3), - Gestationsdiabetes (adjustierte OR = 2,1),

- ungeplante Sectio caesarea (adjustierte $\mathrm{OR}=2,1)$.

\section{Schlussfolgerungen}

Symptome schlafbezogener Atmungsstörungen sind in der Schwangerschaft sehr häufig und assoziiert mit einer höheren Wahrscheinlichkeit des Auftretens von Gestationshypertonie und -diabetes sowie ungeplanter Kaiserschnitte.

\section{Kommentar}

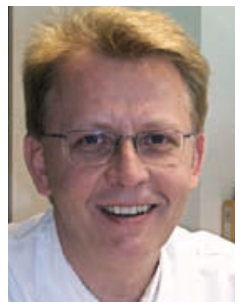

Prof. Dr. med. Kurt Rasche, Wuppertal

Die Studie befasst sich mit einem wichtigen, aber bisher viel zu wenig beachteten Thema, nämlich der Frage, ob schlafbezogene Atmungsstörungen in der Schwangerschaft häufiger sind und ob diese einen Einfluss auf den Schwangerschaftsverlauf sowie das Schicksal des Neugeborenen haben.

Wie pathophysiologisch und aus atemmechanischer Sicht zu erwarten, nehmen Symptome schlafbezogener Atmungsstörungen in der Schwangerschaft zu. Mehr als ein Drittel der Schwangeren schnarcht.

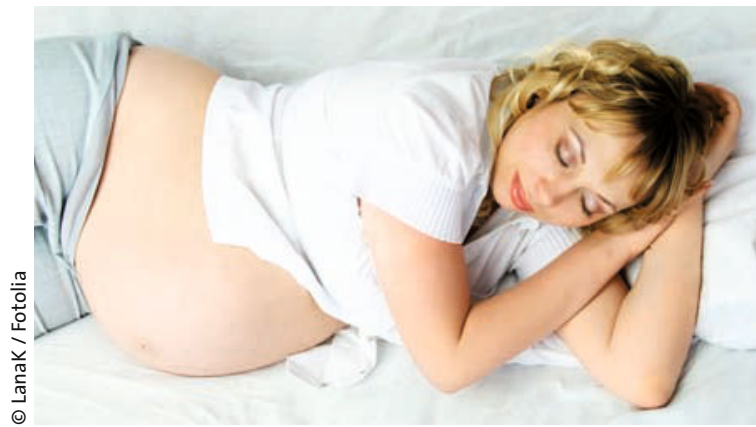

Schwangere Frauen leiden vermehrt unter schlafbezogenen Atmungsstörungen.

Dies ist für die untersuchte Alters- und Geschlechtsgruppe eindeutig erhöht.

Wie auch bei Nichtschwangeren scheinen schlafbezogene Atmungsstörungen einen unmittelbaren fördernden Einfluss auf die Entwicklung einer Gestationshypertonie sowie eines Gestationsdiabetes zu haben. Das relative Risiko steigt hier um das Zweifache. Ähnlich ist dies der Fall im Hinblick auf einen nicht eingeplanten Kaiserschnitt, der indirekt auf eine unmittelbare peripartale fetale Gefährdung hinweist.

Auch wenn die referierte Arbeit einige Limitationen hat, insbesondere die methodische Problematik, dass bei keiner Probandin eine Polygrafie oder gar Polysomnografie angefertigt wurde, sind die Ergebnisse doch äußerst interessant und wichtig, da sie zumindest die Notwendigkeit eines genauen Schlafapnoescreenings in der Schwangerschaft betont. Ob hieraus therapeutische Konsequenzen gezogen werden müssen und ein Einfluss auf den weiteren Verlauf der Schwangerschaft besteht, müssten weitere Studien klären. 